\title{
Epicardial fat thickness and oxidative stress parameters in patients with subclinical hypothyroidism
}

\author{
Ali Aydogdu¹, Emel Yigit Karakas ${ }^{1}$, Emre Erkus², İbrahim Halil Altıparmak², Emin Savık², \\ Turgay Ulas ${ }^{1}$, Tevfik Sabuncu ${ }^{4}$
}

\author{
${ }^{1}$ Department of Internal Medicine, Faculty of Medicine, Harran University, Sanliurfa, \\ Turkey \\ ${ }^{2}$ Department of Cardiology, Faculty of Medicine, Harran University, Sanliurfa, Turkey \\ ${ }^{3}$ Department of Biochemistry, Faculty of Medicine, Harran University, Sanliurfa, Turkey \\ ${ }^{4}$ Department of Internal Medicine and Endocrinology, Faculty of Medicine, Harran \\ University, Sanliurfa, Turkey
}

Submitted: 26 January 2015

Accepted: 23 March 2015

Arch Med Sci 2017; 13, 2: 383-389

DOI: https://doi.org/10.5114/aoms.2017.65479

Copyright (c) 2017 Termedia \& Banach

\begin{abstract}
Introduction: Thyroid disorders are known to be a risk factor for cardiovascular diseases. Epicardial fat thickness (EFT) and oxidative stress are also believed to be major risk factors for cardiovascular events. The aim of this study was to evaluate the possible relationship between oxidative stress parameters and EFT in patients with subclinical hypothyroidism $(\mathrm{SCH})$.

Material and methods: A total of 60 individuals (30 patients with $\mathrm{SCH}$ and 30 healthy controls) were recruited for the study. The EFT and oxidative stress parameters of all participants were analyzed at baseline; the same were analyzed in SCH patients after achievement of a euthyroid state.

Results: Compared to healthy subjects, $\mathrm{SCH}$ patients had significantly higher EFT and oxidative stress parameters ( $p<0.05$ for all). EFT and oxidative stress parameters both decreased after treatment, but only the decrease of EFT levels was statistically significant after thyroid hormone replacement $(p<0.05)$. Serum EFT levels were not significantly correlated with oxidative stress index $(r=0.141, p=0.458)$.

Conclusions: Previous studies have demonstrated that visceral adipose tissue and oxidative stress are major risk factors for cardiovascular events; our study demonstrated that EFT, a visceral adipose tissue, and oxidative stress parameters were higher, and could be used as an indicator for cardiovascular diseases in patients with $\mathrm{SCH}$.
\end{abstract}

Key words: epicardial fat thickness, oxidative stress, subclinical hypothyroidism.

\section{Introduction}

It is well known that thyroid hormones affect the body's metabolism directly. Too little or too much secretion of thyroid hormones changes lipid metabolism, body weight, waist circumference, and body mass in$\operatorname{dex}(\mathrm{BMI})[1]$. Several studies have reported the association between hypothyroidism and metabolic syndrome and hyperlipidemia. Decreasing body fat and non-fat mass were observed in measurements performed by densitometry after the application of hormone replacement therapy to patients with hypothyroidism [2-6]. Several studies have demonstrat-

\author{
Corresponding author: \\ Emel Yigit Karakas \\ Assist. Prof. \\ Department \\ of Internal Medicine \\ Faculty of Medicine \\ Harran University \\ Yenisehir Campus \\ 63300 Sanliurfa, Turkey \\ Phone: +90 5305497361 \\ E-mail: e.ygtkarakas@yahoo. \\ com.tr
}


ed that visceral adipose tissue is a major risk factor for type two diabetes mellitus, dyslipidemia, and cardiovascular events. In addition, epicardial fat thickness (EFT) is an indicator of visceral adipose tissue, and is associated with metabolic syndrome and cardiovascular risk [7-9]. Recent studies have found that products of oxidative stress are responsible for the etiology, pathogenesis, and clinical findings of thyroid disease and cardiovascular disease [10-13].

Although several studies have been performed to discern the relationship of oxidative stress and hypothyroidism, a limited number of studies related to EFT are available on these patients [14-19]. No research study has yet been performed to study the relationship between oxidative stress and EFT in patients with subclinical hypothyroidism $(\mathrm{SCH})$. In order to add to these studies, we examined the relationship between EFT and oxidative stress parameters in patients with $\mathrm{SCH}$, and to examine the effects of levothyroxine treatment on those parameters.

\section{Material and methods}

\section{Study design and patients}

This prospective study was conducted at the Harran University School of Medicine, Sanliurfa, Turkey. Prior to subject recruitment, the study protocol was reviewed and approved by the local ethics committee in accordance with the ethical principles for human investigations, as outlined by the Second Declaration of Helsinki. Written informed consent was obtained from all the patients. From January 2011 to January 2012, 60 consecutive subjects who were admitted to endocrinology and internal medicine polyclinics were recruited for the study.

All study subjects were divided into two groups; group $1(n=30)$ consisted of patients with $\mathrm{SCH}$, and group $2(n=30)$ consisted of healthy subjects. The exclusion criteria were as follows: recent acute infectious illness; any inflammation, infiltrative disorders, or autoimmune diseases; any evidence of liver, kidney, or respiratory disease; diabetes mellitus; uncontrolled essential hypertension; heart failure; malignancy; or regular alcohol use. A detailed history of disease and demographic information was collected from all patients, and all patients underwent physical examination at baseline.

L-thyroxine replacement therapy, with an average daily dose of $1.6 \mu \mathrm{g} / \mathrm{kg}$ body weight for an average 3-6 months, was started for patients who had thyroid-stimulating hormone levels that fell within the upper limit of normal laboratory values of $5.5 \mathrm{mIU} / \mathrm{l}$ (the normal range of thyroid stimulating hormone $(\mathrm{TSH})$ levels $=0.5-5.5 \mathrm{mIU} / \mathrm{l})$, but whose free-T3 (fT3) and free-T4 (fT4) values were within normal values [20]. Blood samples were taken after treatment and stored for the measurement of total oxidant status (TOS), total antioxidant status (TAS), and oxidative stress index (OSI) until the day of analysis.

\section{Baseline definitions and measurements}

Height and weight were measured according to standard protocols. Body mass index (BMI) was calculated as the person's weight in kilograms divided by his or her height in meters squared (kg/ $\mathrm{m}^{2}$ ). Blood pressure was measured using a mechanical sphygmomanometer in the medical office setting. The average of three blood pressure measurements was calculated for each subject after he or she had been seated comfortably for 15 min. Body composition was assessed with bioelectrical impedance analysis (BF 510, Omron Healthcare Co. Ltd., Kyoto, Japan).

\section{Evaluation of blood samples}

All of the blood samples were drawn after $12 \mathrm{~h}$ of overnight fasting from a large antecubital vein without interruption of the venous flow, using a 19-gauge butterfly needle connected to a plastic syringe. Twenty $\mathrm{ml}$ of blood were drawn, with the first few $\mathrm{ml}$ discarded. Ten $\mathrm{ml}$ of blood were used for baseline routine laboratory tests. The residual content of the syringe was transferred immediately to polypropylene tubes, which were then centrifuged at $3,000 \mathrm{rpm}$ for $10 \mathrm{~min}$ at $10-18^{\circ} \mathrm{C}$. Supernatant plasma samples for TAS and TOS were stored in plastic tubes at $-80^{\circ} \mathrm{C}$ after labeling in the biochemistry laboratory until the day of analysis. The TSH, fT3, and fT4 levels were analyzed using an electrochemiluminescence immunometric assay (ECLIA) method with a Roche Elecsys E170 immuno-analyzer (Roche Diagnostics, Burgess Hill, UK). The other biochemical analyses were determined using commercially available assay kits (Abbott, Abbott Park, IL, USA) with an auto-analyzer (Abbott, Abbott Park, IL, USA).

\section{Measurement of oxidative stress parameters}

Serum TOS was measured using a novel automated method developed by Erel [21, 22]. Oxidants present in the sample oxidize the ferrous ion-o-dianisidine complex to ferric ion. The oxidation reaction is enhanced by glycerol molecules, which are abundantly present in the reaction medium. The ferric ion generates a colored complex with xylenol orange in an acidic medium. The color intensity, which can be measured spectrophotometrically (V-530; Jasco, Tokyo, Japan), is related to the quantity of oxidant molecules present in the sample. The assay was calibrated with hydrogen 
peroxide, and the results were expressed in terms of micromolar hydrogen peroxide equivalents per liter ( $\mu \mathrm{mol} \mathrm{H}_{2} \mathrm{O}_{2}$ equiv./l).

Serum TAS was measured using a novel automated method developed by Erel [21, 22]. In this method, the hydroxyl radical, the most potent biological radical, is produced. In the assay, the ferrous ion solution in reagent 1 was mixed with the hydrogen peroxide present in reagent 2 . Sequentially produced radicals, such as the hydroxyl radical-produced brown-colored dianisidinyl radical cation, are also potent radicals. This method allows the measurement of the sample's antioxidative effect against potent free radical reactions that are initiated by the hydroxyl radical. The assay had excellent precision values of more than $97 \%$. The results are expressed as mmol Trolox equiv./l. Trolox equivalent antioxidant capacity measures the antioxidant capacity of a given substance, as compared to the standard, Trolox; as a standard of diphenylpicrylhydrazyl, oxygen radical absorbance capacity and ferric reduce the ability of plasma assays.

The OSI was defined as the ratio of TOS to TAS level. For the calculation, TAS units were changed to $\mathrm{mmol} / \mathrm{l}$, and the OSI value was calculated according to the following formula: OSI (arbitrary unit) $=\operatorname{TOS}\left(\mu \mathrm{mol} \mathrm{H}_{2} \mathrm{O}_{2}\right.$ equiv./l)/TAS (mmol Trolox equiv./l) [21, 22].

\section{Measurement of epicardial fat thickness}

Measurement was performed by a Vivid E9 (General Electric Medical Systems, Milwaukee, Wisconsin, USA) echocardiography device and 2.5 MHz echocardiography probe in the hospital's cardiology department. EFT measurement was performed using a two-dimensional echocardiographic method by transthoracic echocardiography with subjects in the left lateral decubitus position. The EFT was measured on the free wall of the right ventricle from both parasternal longand short-axis views at the mid ventricle during end diastole (marked by the R wave on the ECG recording). The maximum values at each site were measured, and the average value was considered. The measured value was expressed in $\mathrm{cm}$.

\section{Statistical analysis}

Statistical Package for the Social Sciences 20.0 was used for all statistical analyses (SPSS, Chicago, Illinois, USA). The one-sample Kolmogorov-Smirnov test was used to verify the normality of data distributions. Results are expressed as mean \pm SD. The $\chi^{2}$ test was used for categorical variables. The independent sample $t$ test was used to analyze parametric numerical data, and the Mann-Whitney $U$ test was used to analyze non-parametric data. The paired $t$ test for parametric data and the Wilcoxon signed-rank test for non-parametric data were used to analyze changes within the $\mathrm{SCH}$ patients. Pearson correlation coefficients were used to determine correlations between oxidative stress and EFT for patients with $\mathrm{SCH}$. Binary logistic regression analysis was performed to find independent predictors of oxidative stress and EFT in patients with SCH. Values of $p<0.05$ were considered statistically significant for all results.

\section{Results}

The demographic and laboratory parameters of both groups before treatment are shown in Table I. There were no statistically significant differences between the two groups (all $p>0.05$ ) in age, sex, BMI, percent body fat, alanine transaminase, triglycerides, low-density lipoprotein, high-density lipoprotein, total cholesterol, fT3, fT4, systolic blood pressure, or diastolic blood pressure. Compared to the healthy subjects, the $\mathrm{SCH}$ patients had significantly higher TSH, EFT, and OSI levels (all $p<0.05$ ).

Epicardial fat thickness in the SCH patients decreased from the average pre-treatment level of $0.685 \pm 0.237$ to the average level of $0.582 \pm 0.187$ after treatment; this finding is considered statistically significant $(p=0.004)$. There was also a decrease in OSI levels from the average level of 4.78 \pm 1.19 to $4.56 \pm 1.32$, but it was not statistically significant $(p=0.460)$. In addition, there were no differences in TOS and TAS levels before and after treatment $(p>0.05)$ (Table II).

Pearson correlation analysis showed that there was a positive correlation between EFT values and BMI, body fat percent, total cholesterol, low-density lipoprotein, and diastolic blood pressure (all $p<0.05)$. There were no associations with the other parameters (all $p>0.05$ ). In addition, OSI levels were only associated with diastolic blood pressure $(p<0.05)$ (Table III). Binary logistic regression analysis revealed that EFT values were independent predictor for $\mathrm{SCH}$, and OSI levels were an indicator of oxidative stress in $\mathrm{SCH}$ patients $(\beta=4.50$, standard error $=1.68$, Wald statistic $=$ $7.2, p=0.007$; and $\beta=4.24$, standard error $=1.38$, Wald statistic $=9.33, p=0.002$, respectively).

\section{Discussion}

To the best of our knowledge this is the first study to evaluate the correlation between oxidative stress parameters and EFT in patients with $\mathrm{SCH}$. The main findings of the present study were that (i) both EFT and OSI levels were increased in patients with $\mathrm{SCH}$, (ii) but no relationship was found between the two parameters; (iii) EFT and OSI levels decreased after treatment, but only the decrease of EFT levels was statistically significant; (iv) it was observed that EFT values are an indepen- 
Table I. Baseline comparison of the demographic, laboratory and clinical characteristics of all subjects

\begin{tabular}{|c|c|c|c|}
\hline Parameter & SCH patients $(n=30)$ & Healthy subjects $(n=30)$ & $P$-value \\
\hline Age [years] & $37.70 \pm 13.54$ & $40.96 \pm 13.39$ & $N S^{a}$ \\
\hline $\operatorname{Sex}(F / M)$ & $29 / 1$ & $28 / 2$ & $N S^{b}$ \\
\hline $\mathrm{BMI}\left[\mathrm{kg} / \mathrm{m}^{2}\right]$ & $31.81 \pm 8.84$ & $29.20 \pm 6.21$ & $N S^{a}$ \\
\hline Body fat mass (\%) & $41.84 \pm 9.73$ & $37.33 \pm 10.55$ & $N S^{a}$ \\
\hline $\mathrm{ALT}[\mathrm{U} / \mathrm{I}]$ & $18.50 \pm 6.70$ & $23.30 \pm 13.27$ & $\mathrm{NS}^{\mathrm{a}}$ \\
\hline Total cholesterol [mg/dl] & $197.10 \pm 52.87$ & $184.71 \pm 43.62$ & $\mathrm{NS}^{\mathrm{a}}$ \\
\hline LDL cholesterol [mg/dl] & $119.63 \pm 47.17$ & $108.81 \pm 40.55$ & $N S^{a}$ \\
\hline HDL cholesterol [mg/dl] & $46.8 \pm 12.17$ & $45.20 \pm 10.46$ & $N S^{a}$ \\
\hline Triglycerides [mg/dl] & $144.0(45-396)$ & $123.0(57-352)$ & $\mathrm{NS}^{\mathrm{c}}$ \\
\hline $\mathrm{TSH}[\mathrm{mlU} / \mathrm{ll}]$ & $7.52(5.60-21.80)$ & $1.49(0.73-5.34)$ & $<0.001^{c}$ \\
\hline FT3 [pg/ml] & $3.22 \pm 0.46$ & $3.09 \pm 0.40$ & $\mathrm{NS}^{\mathrm{a}}$ \\
\hline $\mathrm{FT} 4$ [ng/dl] & $1.04 \pm 0.16$ & $1.09 \pm 0.15$ & $\mathrm{NS}^{\mathrm{a}}$ \\
\hline Systolic BP [mm Hg] & $122.5(100-150)$ & $125.0(100-140)$ & NSc \\
\hline Diastolic BP [mm Hg] & $80(60-95)$ & $80(70-90)$ & $\mathrm{NS}^{\mathrm{c}}$ \\
\hline $\mathrm{EFT}[\mathrm{cm}]$ & $0.658 \pm 0.237$ & $0.52 \pm 0.16$ & $0.003^{\mathrm{a}}$ \\
\hline TAS [ $\mu \mathrm{mol}$ Trolox equiv./l] & $0.94 \pm 0.16$ & $1.01 \pm 0.12$ & $0.038^{\mathrm{a}}$ \\
\hline $\mathrm{TOS}\left[\mu \mathrm{mol} \mathrm{H}_{2} \mathrm{O}_{2}\right.$ equiv./I] & $44.46 \pm 9.85$ & $23.23 \pm 5.847$ & $<0.001^{a}$ \\
\hline OSI [arbitrary units] & $4.78 \pm 1.19$ & $2.32 \pm 0.59$ & $<0.001^{a}$ \\
\hline
\end{tabular}

Parametric data values are given as mean \pm standard deviation. Nonparametric data values are given as median minimum-maximum. $S C H$ - subclinical hypothyroidism, BMI - body mass index, $A L T$ - alanine transaminase, $L D L$ - low-density lipoprotein, HDL - high-density lipoprotein, TSH - thyroid stimulating hormone, BP - blood pressure, EFT - epicardial fat thickness, TAS - total antioxidant status, TOS - total oxidant status, OSI - oxidative stress index, by independent sample t test ${ }^{a}, \chi^{2 b}$, Mann-Whitney $U$ test $^{c}$.

Table II. Baseline and post-treatment comparisons of hypothyroid patients

\begin{tabular}{|lccc|}
\hline Parameters & Before treatment & After treatment & $P$-value \\
\hline BMI $\left[\mathrm{kg} / \mathrm{m}^{2}\right]$ & $31.81 \pm 8.84$ & $30.96 \pm 8.43$ & $<0.001^{\alpha}$ \\
\hline Body fat mass $(\%)$ & $41.84 \pm 9.73$ & $40.49 \pm 8.54$ & $\mathrm{NS}^{\alpha}$ \\
\hline Total cholesterol $[\mathrm{mg} / \mathrm{dl}]$ & $197.10 \pm 52.87$ & $205.66 \pm 59.34$ & $\mathrm{NS}^{\alpha}$ \\
\hline LDL cholesterol $[\mathrm{mg} / \mathrm{dl}]$ & $119.63 \pm 47.17$ & $133.00 \pm 49.78$ & $\mathrm{NS}^{\alpha}$ \\
\hline HDL cholesterol $[\mathrm{mg} / \mathrm{dl}]$ & $46.80 \pm 12.17$ & $45.96 \pm 12.63$ & $\mathrm{NS}^{\alpha}$ \\
\hline Triglycerides $[\mathrm{mg} / \mathrm{dl}]$ & $144.0(45-396)$ & $112.0(39-330)$ & $\mathrm{NS}^{\beta}$ \\
\hline TSH $[\mathrm{mlU} / \mathrm{ll}]$ & $7.52(5.60-21.80)$ & $2.92(0.57-5.40)$ & $<0.001^{\beta}$ \\
\hline FT3 $[\mathrm{pg} / \mathrm{ml}]$ & $3.22 \pm 0.46$ & $3.16 \pm 0.41$ & $\mathrm{NS}^{\alpha}$ \\
\hline FT4 $[\mathrm{ng} / \mathrm{dl}]$ & $1.04 \pm 0.16$ & $1.18 \pm 0.22$ & $<0.001^{\alpha}$ \\
\hline EFT $[\mathrm{cm}]$ & $0.685 \pm 0.237$ & $0.582 \pm 0.187$ & $0.004^{\alpha}$ \\
\hline TAS $[\mu \mathrm{mol}$ Trolox equiv./l] & $0.94 \pm 0.16$ & $0.97 \pm 0.11$ & $\mathrm{NS}^{\alpha}$ \\
\hline TOS $\left[\mu \mathrm{mol} \mathrm{H} \mathrm{O}_{2}\right.$ equiv./l] & $44.46 \pm 9.85$ & $43.11 \pm 12.77$ & $\mathrm{NS}^{\alpha}$ \\
\hline OSI [arbitrary units] & $4.78 \pm 1.19$ & $4.56 \pm 1.32$ & $\mathrm{NS}^{\alpha}$ \\
\hline
\end{tabular}

Parametric data values are given as mean \pm standard deviation. Nonparametric data values are given as median minimum-maximum. $B M I$ - body mass index, $L D L$ - low-density lipoprotein, HDL - high-density lipoprotein, TSH - thyroid stimulating hormone, EFT-epicardial fat thickness, TAS - total antioxidant status, TOS - total oxidant status, OSI - oxidative stress index; by paired samples test ${ }^{\alpha}$, Wilcoxon signed ranks test ${ }^{\beta}$ 
Table III. Baseline correlation analysis of epicardial fat thickness and oxidative stress index in SCH patients

\begin{tabular}{|c|c|c|c|c|}
\hline \multirow[t]{2}{*}{ Parameter } & \multicolumn{2}{|c|}{ Epicardial fat thickness } & \multicolumn{2}{|c|}{ Oxidative stress index } \\
\hline & $R$ & $P$-value & $R$ & $P$-value \\
\hline Age & 0.464 & 0.10 & 0.130 & 0.493 \\
\hline BMI & 0.530 & $0.003^{*}$ & -0.153 & 0.419 \\
\hline Body fat percent & 0.491 & $0.006^{*}$ & -0.230 & 0.221 \\
\hline ALT & 0.088 & 0.642 & -0.018 & 0.926 \\
\hline Total cholesterol & 0.554 & $0.001^{*}$ & 0.016 & 0.933 \\
\hline LDL cholesterol & 0.542 & $0.002^{*}$ & 0.031 & 0.870 \\
\hline HDL cholesterol & 0.034 & 0.857 & -0.283 & 0.130 \\
\hline Triglycerides & 0.226 & 0.229 & 0.189 & 0.317 \\
\hline TSH & 0.345 & 0.062 & -0.323 & 0.081 \\
\hline FT3 & -0.178 & 0.346 & 0.032 & 0.068 \\
\hline FT4 & -0.276 & 0.140 & 0.009 & 0.964 \\
\hline Systolic BP & 0.173 & 0.361 & 0.259 & 0.167 \\
\hline Diastolic BP & 0.438 & $0.016^{*}$ & 0.466 & $0.009^{*}$ \\
\hline EFT & - & - & -0.141 & 0.458 \\
\hline TAS & 0.012 & 0.951 & -0.490 & $0.006^{*}$ \\
\hline TOS & -0.130 & 0.495 & 0.685 & $<0.001^{*}$ \\
\hline OSI & -0.141 & 0.458 & - & - \\
\hline
\end{tabular}

SCH - subclinical hypothyroidism, BMI - body mass index, ALT - alanine transaminase, $L D L$ - low-density lipoprotein, HDL - high-density lipoprotein, TSH - thyroid stimulating hormone, BP-blood pressure, EFT - epicardial fat thickness, TAS - total antioxidant status, TOS - total oxidant status, OSI - oxidative stress index.

dent predictor for $\mathrm{SCH}$, and OSI levels can be used as an indicator of oxidative stress in $\mathrm{SCH}$ patients.

Several studies have demonstrated the effects of thyroid diseases on oxidative stress. Due to the reduction in thyroid hormone levels that results from hypothyroidism, the metabolic rate slows so that the oxidative products are expected to decrease [23-25]. Conversely, studies have shown that oxidative stress is increased in patients with hypothyroidism [26-28]. Torun et al., Haribabu et al., and Reddy et al. have observed that oxidative stress increased in patients with subclinical and overt hypothyroidism compared to healthy controls [29-31]. Cebeci et al. also found that oxidative stress increased in patients with $\mathrm{SCH}$ compared to controls [32]. In another study, Öztürk et al. observed that oxidative stress increased in patients with overt hypothyroidism, but did not increase in SCH compared to the controls [11]. In our study, oxidative stress parameters were found to be increased in patients with $\mathrm{SCH}$ compared to the controls. There was also a decrease of the levels of OSI after treatment, but this was not found to be statistically significant.

There have been a limited number of studies about the effects of thyroid hormones on EFT. Body weight, body mass index, and waist circumference change if the thyroid hormones are deficient or in excess. Several studies have identified the relationship between $\mathrm{SCH}$ and metabolic syndrome. The EFT is an indicator of visceral adiposity, which is associated with metabolic syndrome and cardiovascular risk. It is more reliable and accurate than waist circumference for measurement of visceral adiposity. Although several studies have demonstrated increased visceral adiposity in $\mathrm{SCH}$, few studies have shown increased EFT in these patients [19, 33-37]. Asik et al. demonstrated that EFT increased in patients with subclinical and overt hypothyroidism compared to the controls [33]. In the current study, EFT increased in patients with SCH compared to the controls, and decreased after the treatment.

No research study in the literature has yet shown the relationship between EFT and oxidative stress in patients with $\mathrm{SCH}$, although the relationship between EFT and oxidative stress has been expressed in a few studies [14-18]. SalgadoSomoza et al. demonstrated that epicardial adipose tissue suffers greater oxidative stress than subcutaneous adipose tissue in patients with cardiovascular diseases; the authors revealed that epicardial adipose tissue may be associated with 
myocardial stress in these patients [15]. Galili et al. found that early abdominal obesity is characterized by increased vascular oxidative stress and endothelial dysfunction in association with increased levels of leptin before the development of insulin resistance and systemic oxidative stress [16]. On the other hand, Wilund et al. demonstrated that intra-dialytic exercise training reduces oxidative stress and EFT [17]. In the current study, we found both increased EFT and OSI levels in $\mathrm{SCH}$ patients compared with the controls.

Although some research studies have investigated the oxidative stress parameters and EFT levels in patients with $\mathrm{SCH}$ separately, these studies did not provide any further results that revealed independent predictors for $\mathrm{SCH}[11,19,29,32,33$, $38,39]$. Only Haribabu et al. have used logistic regression analysis and have revealed that malondialdehyde levels and protein carbonyls levels, both oxidative stress markers, were independent factors for $\mathrm{SCH}$. The authors demonstrated in this study that both malondialdehyde and protein carbonyls levels can be used as markers of oxidative stress in $\mathrm{SCH}$ patients [30]. No knowledge exists in the literature demonstrating, however, that EFT levels are a predictor for SCH. In our study, we further analyzed our results using logistic regression analysis and found that EFT values are an independent predictor for $\mathrm{SCH}$, and that OSI levels can be used as a marker of oxidative stress in $\mathrm{SCH}$ patients, although no positive or negative correlations were found between them.

In conclusion, our present study demonstrated that EFT and OSI levels were higher in patients with $\mathrm{SCH}$ than in healthy subjects. The limitations of the present study are that the sample size was relatively small, and the study was conducted in a single center. Although we have not obtained any results referring to cardiovascular disease another potential limitation of the study - these parameters could be used as an indicator for cardiovascular diseases in patients with $\mathrm{SCH}$, as these levels have been previously proven to be related to cardiovascular diseases. Therefore, there is a need for multicenter, prospective, randomized studies in which a greater number of patients are studied in order to confirm this topic.

\section{Conflict of interest}

The authors declare no conflict of interest.

\section{References}

1. Ulas T, Buyukhatipoglu H, Eren MA, et al. Evaluation of sleeping energy expenditure using the SenseWear Armband in patients with overt and subclinical hypothyroidism. Clin Invest Med 2012; 35: E126-31.

2. Erdogan M, Canataroglu A, Ganidagli S, Kulaksızoglu M. Metabolic syndrome prevalence in subclinic and overt hypothyroid patients and the relation among metabolic syndrome parameters. J Endocrinol Invest 2011; 34: 488-92.

3. Lee YK, Kim JE, Oh HJ, et al. Serum TSH level in healthy Koreans and the association of TSH with serum lipid concentration and metabolic syndrome. Korean J Intern Med 2011; 26: 432-9.

4. Waring AC, Rodondi N, Harrison S, et al. Thyroid function and prevalent and incident metabolic syndrome in older adults: the health, aging, and body composition study. Clin Endocrinol (Oxf) 2012; 76: 911-8.

5. Aghili R, Khamseh ME, Malek M, et al. Changes of subtests of Wechsler Memory Scale and cognitive function in subjects with subclinical hypothyroidism following treatment with levothyroxine. Arch Med Sci 2012; 8: 1096-101.

6. Aronow WS. Peripheral arterial disease of the lower extremities. Arch Med Sci 2012; 8: 375-88.

7. Lemieux S. Contribution of visceral obesity to the insulin resistance syndrome. Can J Appl Physiol 2001; 26: 273-90.

8. Liao Y, Kwon S, Shaughnessy S, et al. Critical evaluation of adult treatment Panel III criteria in identfying insulin resistance with dyslipidemia. Diabetes Care 2004; 27: 978-83.

9. Iacobellis G, Ribaudo MC, Assael F, et al. Echocardiographic epicardial adipose tissue is related to anthropometric and clinical parameters of metabolic syndrome: a new indicator of cardiovascular risk. J Clin Endocrinol Metab 2003; 88: 5163-8.

10. Bianchi G, Solaroli E, Zaccheroni V, et al. Oxidative stress and anti-oxidant metabolites in patients with hyperthyroidism: effect of treatment. Horm Metab Res 1999; 31: 620-4.

11. Öztürk Ü, Vural P, Özderya A, Karadağ B, Doğru-Abbasoğlu S, Uysal M. Oxidative stress parameters in serum and low density lipoproteins of Hashimoto's thyroiditis patients with subclinical and overt hypothyroidism. Int Immunopharmacol 2012; 14: 349-52.

12. Ulas T, Tursun I, Demir ME, Dal MS, Buyukhatipoglu $H$. Comment on: infusion of lin-/sca-1+ and endothelial progenitor cells improves proinflammatory and oxidative stress markers in atherosclerotic mice. Int J Cardiol 2013; 164: 128.

13. Ulas T, Hacıbekiroglu T, Sezen $H$, Buyukhatipoglu $H$. Comment on: analysis of oxidative stress expressed by urinary level of 8-hydroxy-2'-deoxyguanosine and biopyrrin in atrial fibrillation: effect of sinus rhythm restoration. Int J Cardiol 2013; 167: 1643.

14. Lin YK, Chen YJ, Chen SA. Potential atrial arrhythmogenicity of adipocytes: implications for the genesis of atrial fibrillation. Med Hypotheses 2010; 74: 1026-9.

15. Salgado-Somoza A, Teijeira-Fernández E, Fernández AL, González-Juanatey JR, Eiras S. Proteomic analysis of epicardial and subcutaneous adipose tissue reveals differences in proteins involved in oxidative stress. Am J Physiol Heart Circ Physiol 2010; 299: H202-9.

16. Galili O, Versari D, Sattler KJ, et al. Early experimental obesity is associated with coronary endothelial dysfunction and oxidative stress. Am J Physiol Heart Circ Physiol 2007; 292: H904-11.

17. Wilund KR, Tomayko EJ, Wu PT, et al. Intradialytic exercise training reduces oxidative stress and epicardial fat: a pilot study. Nephrol Dial Transplant 2010; 25: 2695-701.

18. Sacks HS, Fain JN, Cheema P, et al. Depot-specific overexpression of proinflammatory, redox, endothelial cell, and angiogenic genes in epicardial fat adjacent to se- 
vere stable coronary atherosclerosis. Metab Syndr Relat Disord 2011; 9: 433-9.

19. Unubol M, Eryilmaz U, Guney E, Akgullu C, Kurt Omurlu I. Epicardial adipose tissue in patients with subclinical hypothyroidism. Minerva Endocrinol 2014; 39: 135-40.

20. So M, Maclsaac RJ, Grossmann M. Hypothyroidism. Aust Fam Physician 2012; 41: 556-62.

21. Erel O. A novel automated method to measure total antioxidant response against potent free radical reactions. Clin Biochem 2004; 37: 112-9.

22. Erel O. A new automated colorimetric method for measuring total oxidant status. Clin Biochem 2005; 38: 1103-11.

23. Pereira B, Rosa LF, Safi DA, Bechara EJ, Curi R. Control of superoxide dismutase, catalase and glutathione peroxidase activities in rat lymphoid organs by thyroid hormones. J Endocrinol 1994; 140: 73-7.

24. Swaroop A, Ramasarma T. Heat exposure and hypothyroid conditions decrease hydrogen peroxide generation in liver mitochondria. Biochem J 1985; 226: 403-8.

25. Oziol L, Faure P, Bertrand N, Chomard P. Inhibition of in vitro macrophage-induced low density lipoprotein oxidation by thyroid compounds. J Endocrinol 2003; 177: 137-46.

26. Yilmaz S, Ozan S, Benzer F, Canatan H. Oxidative damage and antioxidant enzyme activities in experimental hypothyroidism. Cell Biochem Funct 2003; 21: 325-30.

27. Dumitriu L, Bartoc R, Ursu H, Purice M, Ionescu V. Significance of high levels of serum malonyl dialdehyde (MDA) and ceruloplasmin (CP) in hyper- and hypothyroidism. Endocrinologie 1988; 26: 35-8.

28. Costantini F, Pierdomenico SD, De Cesare D, et al. A. Effect of thyroid function on LDL oxidation. Arterioscler Thromb Vasc Biol 1998; 18: 732-7.

29. Torun AN, Kulaksizoglu S, Kulaksizoglu M, Pamuk BO, Isbilen E, Tutuncu NB. Serum total antioxidant status and lipid peroxidation marker malondialdehyde levels in overt and subclinical hypothyroidism. Clin Endocrinol (Oxf) 2009; 70: 469-74.

30. Haribabu A, Reddy VS, Pallavi C, et al. Evaluation of protein oxidation and its association with lipid peroxidation and thyrotropin levels in overt and subclinical hypothyroidism. Endocrine 2013; 44: 152-7.

31. Reddy VS, Gouroju S, Suchitra MM, et al. Antioxidant defense in overt and subclinical hypothyroidism. Horm Metab Res 2013; 45: 754-8.

32. Cebeci E, Oner FA, Usta M, Yurdakul S, Erguney M. Evaluation of oxidative stress, the activities of paraoxonase and arylesterase in patients with subclinic hypothyroidism. Acta Biomed 2011; 82: 214-22.

33. Asik M, Sahin S, Ozkul F, et al. Evaluation of epicardial fat tissue thickness in patients with Hashimoto thyroiditis. Clin Endocrinol (Oxf) 2013; 79: 571-6.

34. Biondi B, Cooper DS. The clinical significance of subclinical thyroid dysfunction. Endocr Rev 2008; 29: 76-131.

35. lacobellis G, Sharma AM. Epicardial adipose tissue as new cardio-metabolic risk marker and potential therapeutic target in the metabolic syndrome. Curr Pharm Des 2007; 13: 2180-4

36. Iacobellis G, Assael F, Ribaudo MC, et al. Epicardial fat from echocardiography: a new method for visceral adipose tissue prediction. Obes Res 2003; 11: 304-10.

37. Korkmaz L, Sahin S, Akyuz AR, et al. Epicardial adipose tissue increased in patients with newly diagnosed subclinical hypothyroidism. Med Princ Pract 2013; 22: 42-6.

38. Santos OC, Silva NA, Vaisman M, et al. Evaluation of epicardial fat tissue thickness as a marker of cardiovas- cular risk in patients with subclinical hypothyroidism. J Endocrinol Invest 2015; 38: 421-7.

39. Santi A, Duarte MM, de Menezes CC, Loro VL. Association of lipids with oxidative stress biomarkers in subclinical hypothyroidism. Int J Endocrinol 2012; 2012: 856359. 